\title{
Numerical Investigation of Well-Structured Porous Media in a Milli-Scale
}

\section{Tubular Reactor}

M. Mottaghi, S. Kuhn

KU Leuven, Department of Chemical Engineering, 3001 Leuven, Belgium

\begin{abstract}
Despite the many advantages of micro-reactors their application in industry is still limited due to their low throughput. To address this issue, micro-scale porous media are used as inserts in milli-scale tubular reactors. In this work, six different well-structured porous media (WSPM) consisting of cylindrical rods with an outer diameter of $250 \mu \mathrm{m}$ are characterized with computational fluid dynamics (CFD). The individual WSPM differ in the rotation of rod layers around the main flow direction, and the orientation of rod layers against the flow direction. The simulation results show that changing the rod layer orientation influences the flow heterogeneity, while the rod layer rotation affects the pore alignment resulting in connected fluid paths. Scalar transport simulations reveal that the WSPM result in a considerable mixing enhancement. This study underlines the potential of integrating WSPM in milli-scale reactors as scale-up approach, and how their geometry affects mixing.
\end{abstract}

\section{Keywords:}

milli-scale reactor; porous medium, CFD, mixing, scale-up

\section{Introduction}

The efforts in process intensification, e.g. to manufacture chemicals in a more sustainable fashion (Suranani et al. 2018), led to the development of various novel approaches for continuous reaction systems (Zhang et al. 2017). One of these approaches is the use of highly 
porous media as inserts for flow reactors (Razza et al. 2016) as they provide a large specific surface area with associated low pressure drop (Hall \& Hiatt 1994)(Lämmermann et al. 2018). In general, highly porous media can be classified in two main groups based on their geometry, which are stochastic structures (e.g. random open-cell foams) and ordered structures (Huu et al. 2009). Early studies revealed the potential of using random open-cell foams as packing material in flow reactors. Using cartridges of metallic or ceramic foams as heterogeneous catalyst was observed to increase the reaction yield of the catalytic methane combustion (Podyacheva et al. 1997), the production of hydrogen via the water-gas shift reaction (Lang et al. 2017) and carbon dioxide reforming (Twigg \& Richardson 2007). These improvements are linked to the large surface-to-volume ratio and tortuous flow paths in these random open-cell foams (Giani et al. 2005a; Giani et al. 2005b). In addition, their low density, high porosity and high mechanical strength make them a promising alternative to the commonly used packed beds. The porosity, or cell size, of random open-cell foams is expressed in terms of pores per inch (PPI), which has been varied as the main parameter to study the effect of random open-cell foams in flow reactors (Boomsma \& Poulikakos 2002). Hutter et al observed that inserting metal foams in the 20-45 PPI range into a tubular reactor of $7 \mathrm{~mm}$ inner diameter significantly improves mass transfer and mixing compared to an empty tube in single phase flow. It was also found that the metal foam of 20 PPI exhibits better mixing in comparison with the metal foam of 30 PPI at high Reynolds numbers (Re>600 based on bulk velocity and tube diameter) as its increased strut thickness induces a higher turbulence intensity. Furthermore, using the metal foam of 45 PPI increases the pressure drop because of its smaller pore sizes (Hutter et al. 2010; Hutter et al. 2011a).

Tourvieille et al utilized metal foams in the 35-55 PPI range to study their effects on two phase gas-liquid flow in a square channel of $2 \times 2 \mathrm{~mm}^{2}$. It was found that the foam with the higher PPI results in the largest pressure drop, but also exhibits a higher overall mass transfer coefficient (Tourvieille et al. 2015b; Tourvieille et al. 2015a). Serres et al compared gas-liquid flow in 
reactors equipped with a micro-scale packed bed and metal foams of different PPI, and they observed that the metal foam with $80 \mathrm{PPI}$ and the micro-scale packed bed have nearly the same liquid holdup (Serres et al. 2016; Serres et al. 2018).

However, the primary challenge associated with random open-cell foams is that their particular geometry is solely described by a single parameter, i.e. their PPI, which renders the generalization of correlations to predict e.g. pressure drop, permeability and mass transfer coefficients difficult (Lacroix et al. 2007; Razza et al. 2016; Klumpp et al. 2014). Therefore, the use of ordered structures is essential to systematically study the effect of their geometrical parameters in flow reactors (Busse et al. 2018; Inayat et al. 2011). In that regard, the most widely studied ordered porous structure is the Kelvin-like unit cell, a polyhedron unit cell with pentagonal or hexagonal faces (Huu et al. 2009). Lammerman et al reported that modifying the Kelvin-like unit cell by combining it with a Diamond-like unit cell results in a more uniform liquid distribution in gas-liquid flow (Lämmermann et al. 2016). Furthermore, increasing the window size of this hybrid unit cell decreases the dynamic liquid holdup (Lämmermann et al. 2018). The challenge of utilizing the Kelvin-like unit cell as porous insert in flow reactors is to develop analytical models to calculate their ligament surface area and porosity, which is needed in correlations to predict pressure drop and mass transfer coefficients (Kumar \& Topin 2014; Della Torre et al. 2016).

Another approach to define a structured unit cell is to subtract a close-packing of spheres from a volume, which was applied as well-structured porous media (WSPM) in milli-tubular reactors (Hutter et al. 2010; Hutter et al. 2011a; Krishnan et al. 2006). Hutter et al observed that these WSPM have similar axial dispersion coefficients as 20 and 30 PPI metal foams (Hutter et al. 2011b). Häfeli et al applied these WSPM to the emulsification of anisole and a brine, and it was found that the droplet size generated with the WSPM is smaller than the droplets generated with a SMX mixer (Häfeli et al. 2016). The numerical study by Hutter et al highlights the effect of the ligament shape on the WSPM hydrodynamics. Ligaments with sharp edges induced an up 
to $30 \%$ higher turbulent kinetic energy and pressure drop in contrast to round ligaments (Hutter et al. 2011b).

WSPM can also be constructed based on layers of cylindrical rods (Potdar et al. 2017; Potdar et al. 2019a; Potdar et al. 2019b), which then allows for additional degrees of freedom to construct the pore network, as individual rod layers can be freely shifted and rotated against each other. Introducing a rotation will alter the alignment of the unit cell windows and the connectivity of individual pores, contrary to WSPM based on sphere-like or Kelvin-like unit cells, in which the spatial alignment is fixed. These advantages make WSPM based on microscale cylindrical rods an interesting option to be utilized in milli-scale tubular reactors.

Therefore, in this work, we study the effect of various configurations of aforementioned WSPM embedded in a milli-tubular reactor on the flow hydrodynamics and the mixing by using computational fluid dynamics (CFD). The flow hydrodynamics are analyzed by quantifying the flow number (Yang \& Manas-Zloczower 1992), stretching efficiency (Ottino 1989) and the finite-time Lyapunov exponent (FTLE) (Haller 2001), whereas the axial and radial mixing is characterized with a RTD analysis (Levenspiel 1999) and computing the intensity of segregation (Danckwerts 1952). The combination of these methods allows to link the flow behavior and mixing in the WSPM to its geometrical properties.

\section{Geometrical details of the WSPM}

In this work, six different porous reactors are investigated, where the WSPM consists of cylindrical rods with an outer diameter of $250 \mu \mathrm{m}$ arranged in stacks in consecutive layers (see Figure 1). Common to all structures is that the consecutive layers are shifted relatively to each other by a third of the inter-rod distance (see Figure 1a). Furthermore, the rod layers are rotated by an angle $\theta$ around the main flow direction (z-axis, Figure 1b) and oriented with an angle $\phi$ towards the plane normal to the main flow direction (x-y plane, Figure 1c). The values of each angle are represented in the naming convention for each structure, i.e. the structure $S \_22.5 t \_45 p$ 
features values of $\theta=22.5^{\circ}$ and $\phi=45^{\circ}$, see Table 1 for the geometrical details of each porous reactor. The WSPM are embedded into tubes with an inner diameter of $D=3.4 \mathrm{~mm}$ and a length of $7 \mathrm{~cm}$ (see Figure 2). Potdar et al. (2017) experimentally investigated the porous reactors S_0t_0p and S_22.5t_45p, and it was observed that the structure S_22.5t_45p had a significantly improved mass transfer performance, hence the parametric study of $\theta$ and $\phi$ in this work is based on these values.

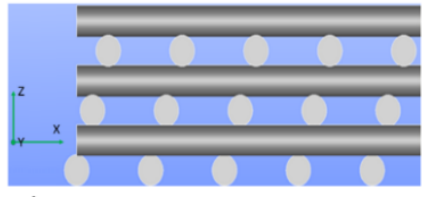

a)

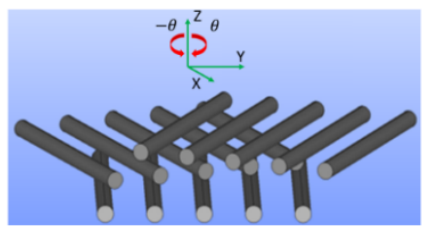

b)

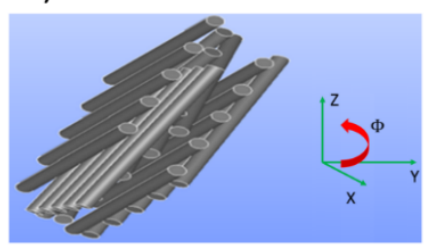

c)

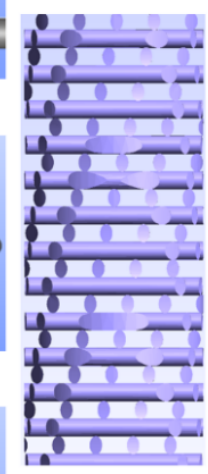

d)

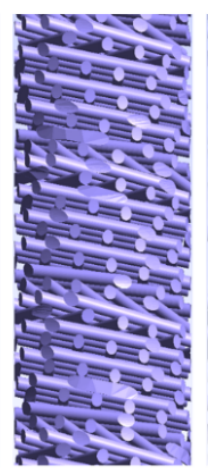

e)

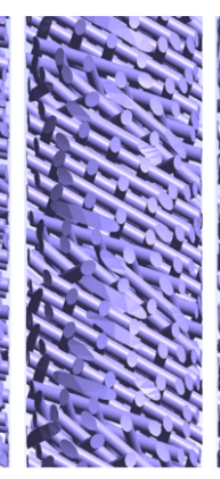

f)

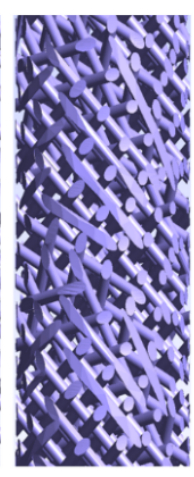

g)

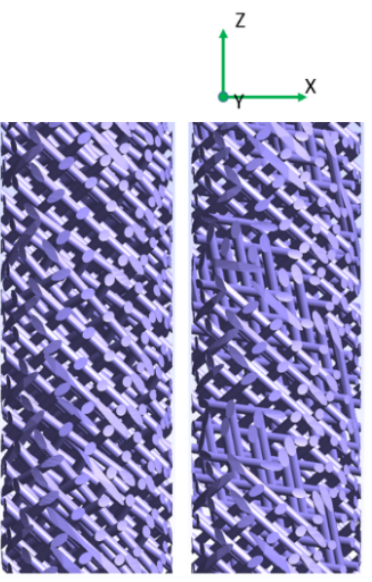

h)$$
\text { i) }
$$

Figure 1 Geometrical details of the well-structured porous reactors build from cylindrical rods:

a) Consecutive layers are shifted relatively to each other by a third of the inter-rod distance;

b) Rotation of rod layers by an angle $\theta$ around the main flow direction (z-axis); c) Orientation of rod layers with an angle $\phi$ towards the plane normal to the main flow direction (x-y plane); d) WSPM S_0t_0p ; e) WSPM S_22.5t_9p; f) WSPM S_22.5t_22.5p; g) WSPM S_22.5t_45p; h) WSPMS_9t_45p; i) WSPM S_45t_45p. 
Table 1 Geometrical details of the well-structured porous reactors.

\begin{tabular}{|c|c|c|c|c|c|}
\hline & & Specific & & & Void volume \\
\hline Structure & Porosity & $\begin{array}{l}\text { surface area } \\
\left(\mathrm{m}^{2} \mathrm{~m}^{-3}\right)\end{array}$ & $\theta\left(^{\circ}\right)$ & $\Phi\left(^{\circ}\right)$ & $\mathrm{x} 10^{7}\left(\mathrm{~m}^{3}\right)$ \\
\hline S_0t_0p & $72 \%$ & 4480 & $\theta=0.0$ & $\Phi=0.0$ & 4.48 \\
\hline S_22.5t_9p & $73 \%$ & 4240 & $\theta=22.5$ & $\Phi=9.0$ & 4.54 \\
\hline $\mathrm{S} \_22.5 \mathrm{t} \_22.5 \mathrm{p}$ & $73 \%$ & 4230 & $\theta=22.5$ & $\Phi=22.5$ & 4.55 \\
\hline $\mathrm{S} \_22.5 \mathrm{t} \_45 \mathrm{p}$ & $74 \%$ & 4160 & $\theta=22.5$ & $\Phi=45.0$ & 4.6 \\
\hline S_9t_45p & $74 \%$ & 4155 & $\theta=9.0$ & $\Phi=45.0$ & 4.6 \\
\hline S_45t_45p & $74 \%$ & 4165 & $\theta=45.0$ & $\Phi=45.0$ & 4.61 \\
\hline
\end{tabular}

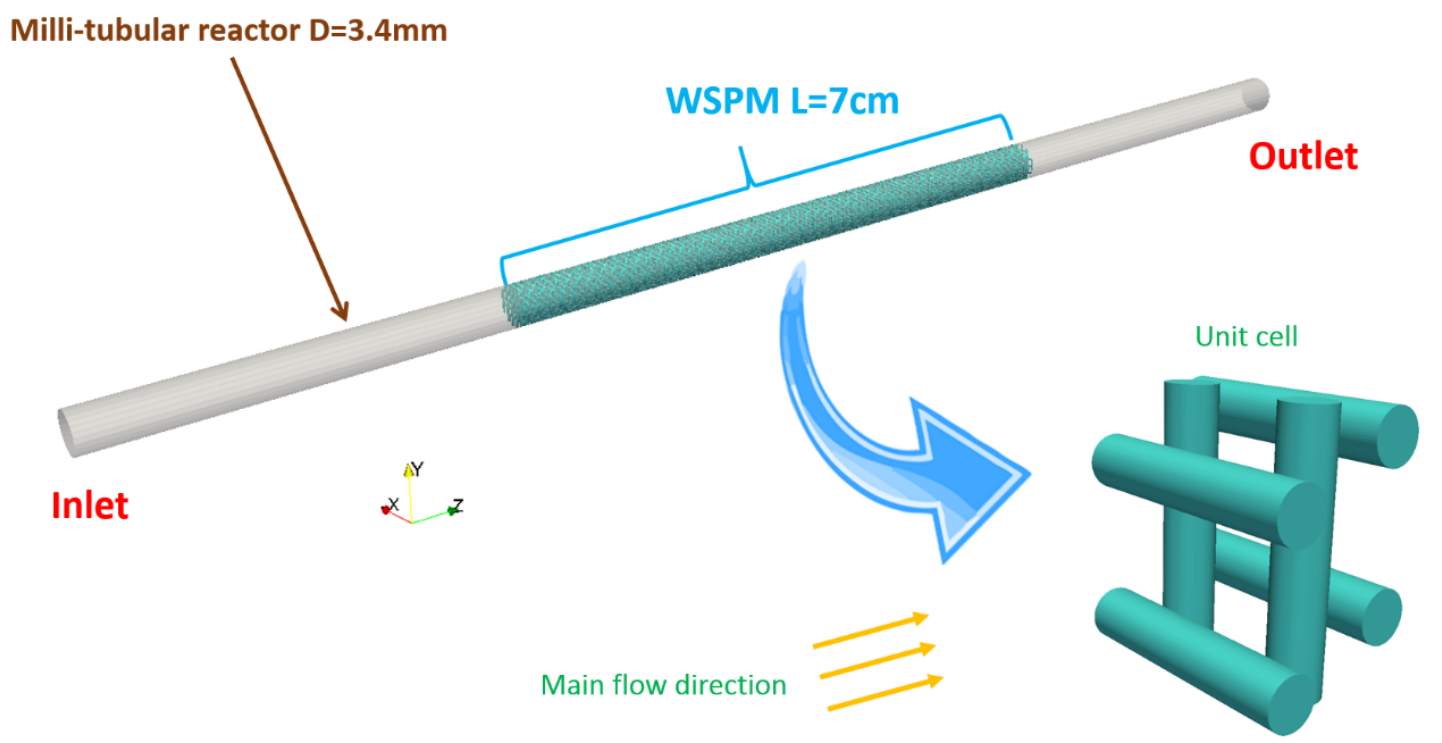

Figure 2 Schematic representation of the WSPM inserted in a milli-tubular reactor with a magnified example of a unit cell shape.

The geometry of each structure was prepared in Salome-Meca (Code_aster 2018) using the built-in python interface, which was then subsequently meshed using snappyHexMesh in OpenFOAM 3.0.x. A structured mesh has been chosen as it provides more efficiency, faster convergence and better numerical performance due to less numerical diffusion compared to 
unstructured grids (Versteeg \& Malalasekera 2007). Three different mesh resolutions per geometry have been generated to study the mesh independence of the results (see Table 2).

Table 2 Total number of computational cells and the characteristic cell size for the three different meshes used in this work.

\begin{tabular}{lll}
\hline $\begin{array}{l}\text { Mesh resolution } \\
(\text { million cells) }\end{array}$ & $\begin{array}{ll}\text { Near-wall cell } \\
\text { dimension }(\mu \mathrm{m})\end{array}$ & Pore cell dimension $(\mu \mathrm{m})$ \\
\hline $5-6$ & 7.5 & 40 \\
$11-12$ & 3 & 30 \\
$20-22$ & 1.5 & 15 \\
\hline
\end{tabular}

Figure $3 \mathrm{a}$ depicts the obtained axial velocity profiles from the $\mathrm{S}_{-} 22.5 \mathrm{t} \_45 \mathrm{p}$ reactor at a downstream position of $0.9 L$, where $L$ represents the porous structure length, for the three different mesh resolutions. Only negligible differences in the velocity profiles are observed when increasing the mesh resolution. Furthermore, the calculated mixing efficiency (OrtegaCasanova 2017; Rahimi et al. 2015) from the three mesh resolutions of the same structure also exhibit only minor differences, see Figure 3b. Hence, the resolution of 5-6 million cells was chosen for the remainder of this work to limit the computational time. 
a)

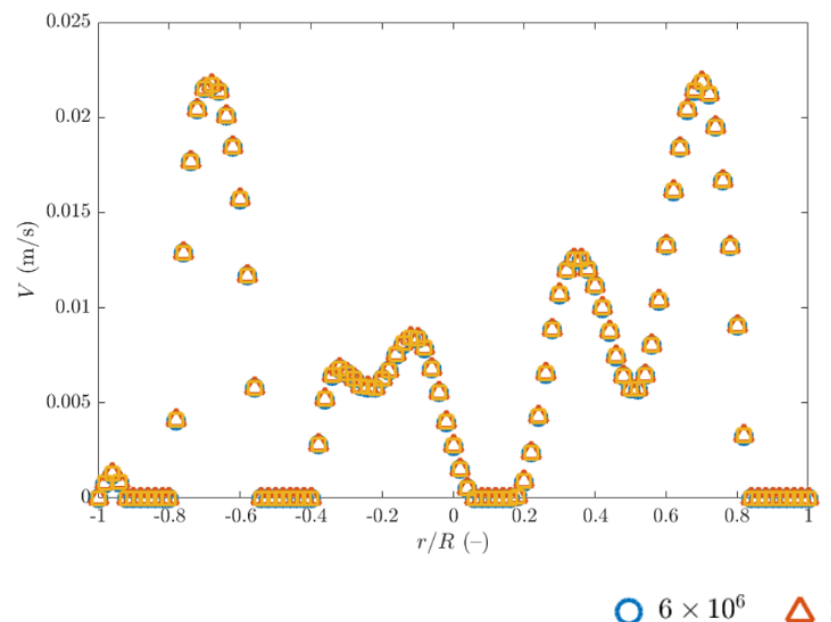

b)

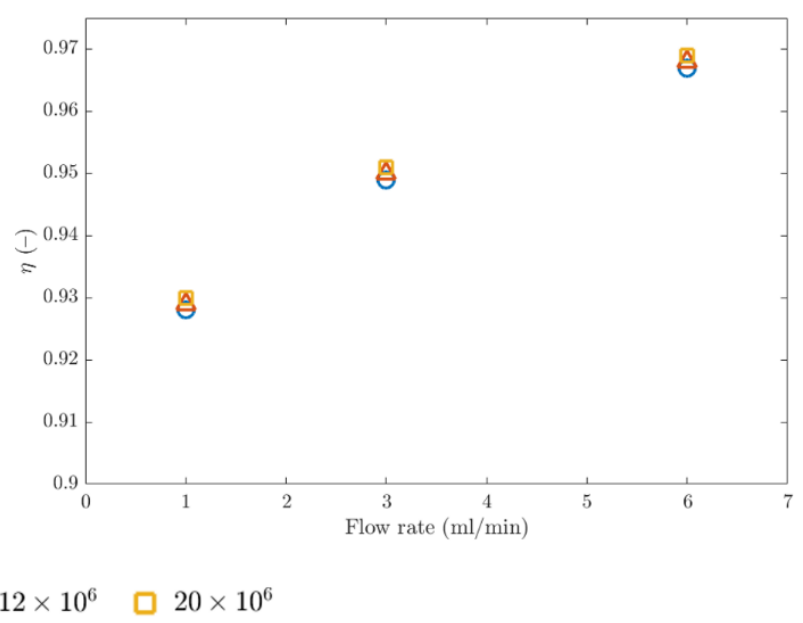

Figure 3 a) Axial velocity profiles within the $S \_22.5 t+45 p$ reactor at the axial position $z=0.9 L$ for a total flow rate $3 \mathrm{ml} / \mathrm{min}$ and three mesh resolutions 6 million cells (circle), 12 million cells (triangle) and 20 million cells (square); b) the mixing efficiency $\left(\eta=1-\sqrt{\frac{\sigma^{2}}{\sigma_{\max }^{2}}}\right)$ of the S_22.5t_45p reactor subject to the flow rate $1 \mathrm{mil} / \mathrm{min}, 3 \mathrm{ml} / \mathrm{min}$ and $6 \mathrm{ml} / \mathrm{min}$ for three mesh resolutions 6 million cells (circle), 12 million cells (triangle) and 20 million cells (square).

\section{Numerical methods}

Simulations have been carried out for the three flow rates of $1 \mathrm{ml} / \mathrm{min}, 3 \mathrm{ml} / \mathrm{min}$, and $6 \mathrm{ml} / \mathrm{min}$, corresponding to the empty tube based Reynolds numbers of 7, 21 and 41 respectively. The obtained pore based Reynolds numbers also show low values $(\mathrm{Re}<5)$ in the selected flow rate range, and therefore laminar flow can be assumed in the entire porous structure (Della Torre et al. 2014). The flow is governed by the incompressible Navier-Stokes equations for the conservation of mass and momentum

$$
\begin{gathered}
\nabla \cdot \vec{V}=0 \\
\frac{\partial \vec{V}}{\partial t}+\vec{V} \cdot \nabla \vec{V}=-\frac{1}{\rho} \nabla p+\nu \nabla^{2} \vec{V}
\end{gathered}
$$

where $\vec{V}$ is the velocity vector, $p$ denotes the pressure, $\rho$ the density, and $v$ the kinematic viscosity. For the simulations water has been used as the fluid medium with a density of 
$998 \mathrm{~kg} / \mathrm{m}^{3}$ and a dynamic viscosity of $8.9 \cdot 10^{-4} \mathrm{~Pa} \cdot \mathrm{s}$. The concentration field of a species is determined by solving the convection-diffusion transport equation

$$
\frac{\partial C_{A}}{\partial t}+(\vec{V} \cdot \nabla) C_{A}=D_{A} \nabla^{2} C_{A}
$$

where $C_{A}$ is the species concentration and $D_{A}$ represents its diffusivity. The physical properties of water were used for the dye solution with the tracer diffusivity of $D_{A}=1.0 \cdot 10^{-10} \mathrm{~m}^{2} / \mathrm{s}$ (Woldemariam et al. 2016).

These equations are solved using the finite volume based OpenFOAM ${ }^{\circledR}-3.0 . x$ software package (Weller 2018). Three solvers were employed in this study: The incompressible Navier-Stokes equations (Eqs. $1 \& 2$ ) are computed by using either the simpleFoam (steady-state) or the pimpleFoam (transient) solver. To ensure stable computations, a relaxation factor of 0.9 was applied for velocity and pressure. The resulting flow field is then used to solve the species transport equation (Eq. 3) with the scalarTransportFoam solver. In these simulations, a volume of tracer is injected $0.5 \mathrm{~mm}$ upstream of the porous structure at $t=0 \mathrm{~s}$, see Figure 4 .

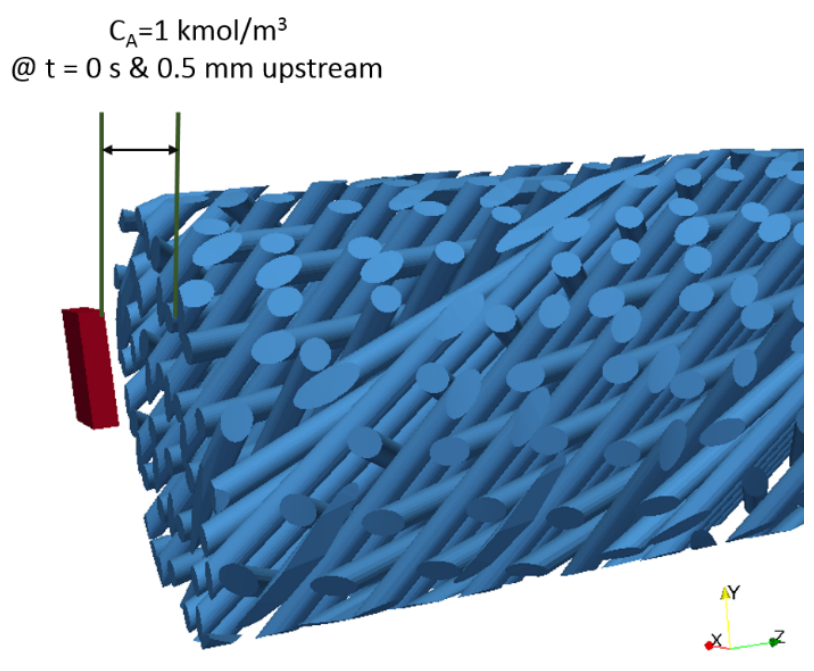

Figure 4 Schematic of the tracer injection $0.5 \mathrm{~mm}$ upstream in one quadrant of the porous structure at $t=0$ s to facilitate the scalarTransportFoam simulations. 
Section S1 in the Supplementary Information provides details on the numerical schemes used in the simulations, and the applied boundary conditions are summarized in Table 3.

Table 3 Boundary conditions for pressure, velocity, and concentration.

\begin{tabular}{lcll}
\hline Boundaries & Pressure & Velocity & Concentration \\
\hline Inlet & Zero gradient & $\vec{V}=0.0054 \mathrm{~m} / \mathrm{s}$ & $C_{A}=0 \mathrm{~mol} / \mathrm{m}^{3}$ \\
Outlet & $P=0 \mathrm{~Pa}$ & Zero gradient & Zero gradient \\
Walls & Zero gradient & Zero velocity & Zero gradient \\
\hline
\end{tabular}

\section{Results and Discussion}

\subsection{Effect of Structure on the Flow Field}

\subsubsection{Radial and Axial Velocity Profiles}

Contours of the radial and axial velocity in the $y, z$-plane of all structures are provided in Figure 5. It is observed that for the structure $\mathrm{S}_{-} 0 \mathrm{t} \_0 \mathrm{p}$, the fluid is uniformly distributed radially, i.e. solid elements split and recombine the fluid elements (Harnby et al. 1997). Increasing the orientation angle $\phi$ of the porous structure against the plane normal to the flow direction (zaxis) up to a value of $45^{\circ}$ ( $\left.\mathrm{S} \_22.5 \mathrm{t} \_45 \mathrm{p}\right)$ significantly affects the radial velocity profiles as a more heterogeneous distribution is generated. Interactions between these heterogeneous regions with different radial velocity magnitudes results in increasing the shear in the plane normal to the main flow direction (Harnby et al. 1997). Furthermore, changes in the orientation angle $\phi$ affect the distribution of the axial velocity, as shorter connected fluid paths are established. The influence of the rotation angle $\theta$ on the radial and axial velocities is less pronounced for $9^{\circ}$ and $22.5^{\circ}$, but further increasing it to $45^{\circ}$ results in longer connected fluid paths. 
a)
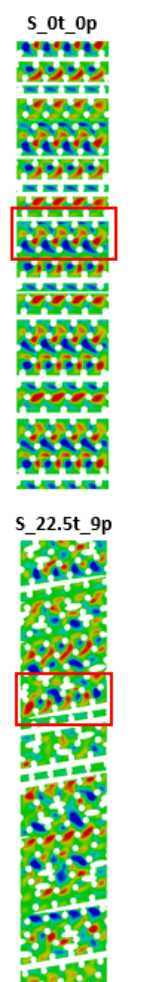

S 9 9t $45 p$

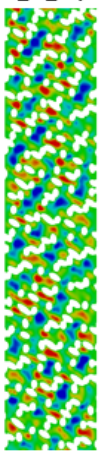

Ucyl rad
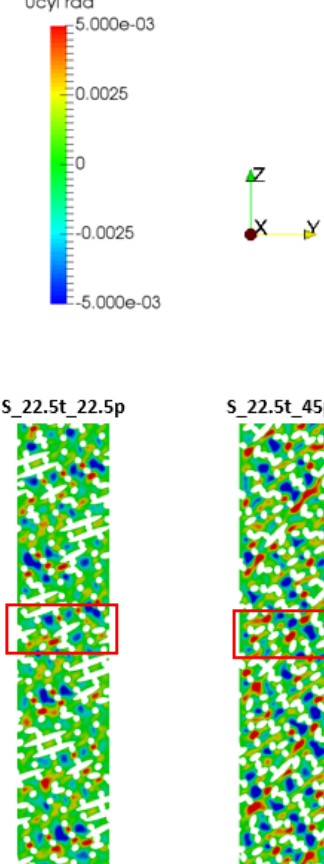

S $22.5 \mathrm{t}$ 45p

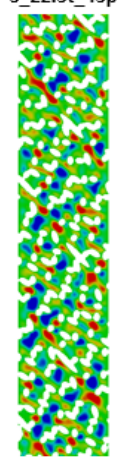

S_-45t $45 p$ b)
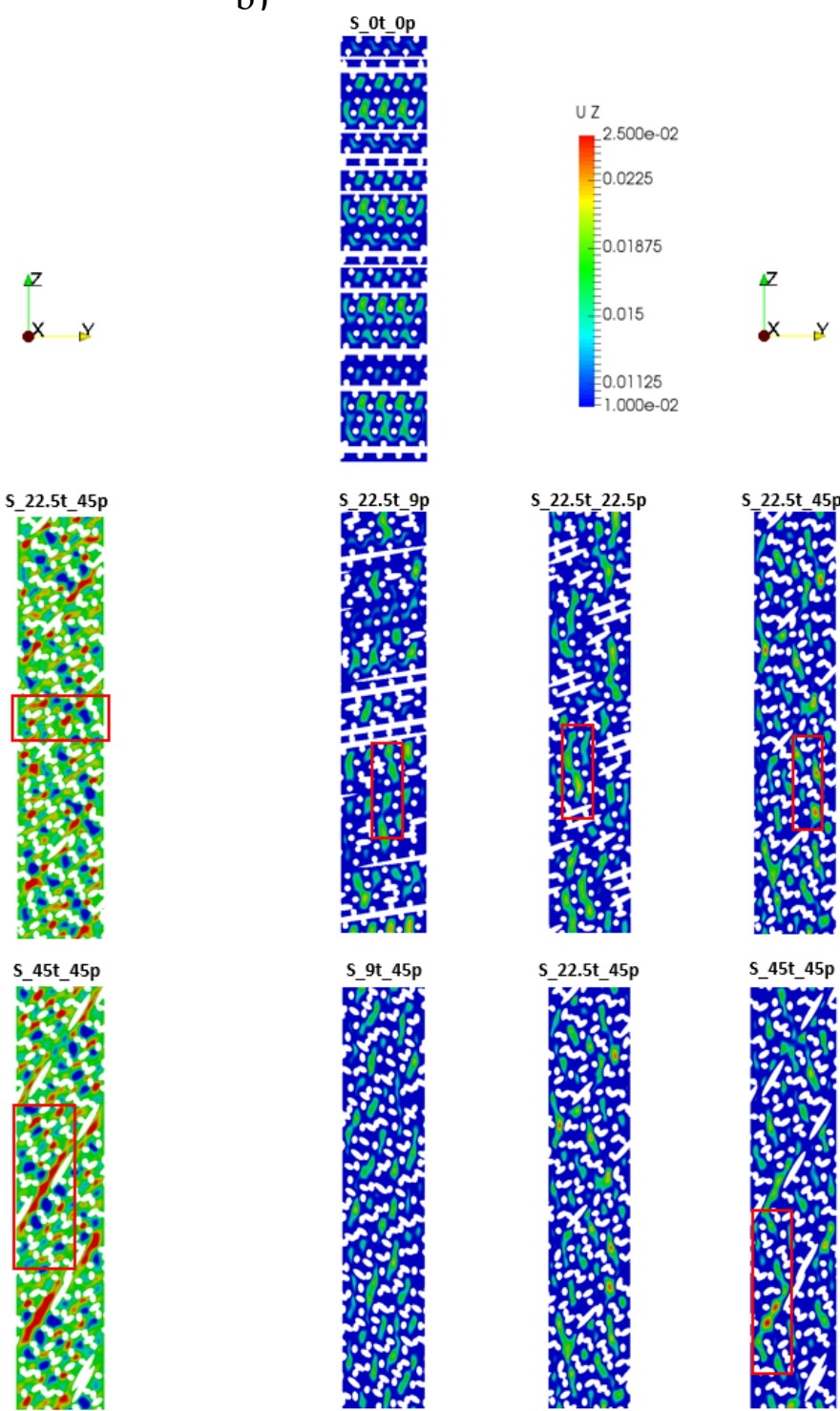

S 9 t $45 p$

S_22.5t_45p

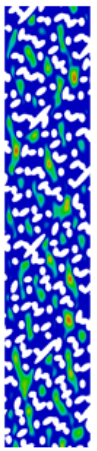

S_ 45 t $45 p$

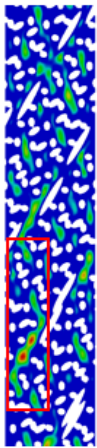

Figure 5 (a) Radial and (b) axial velocity contours for a flow rate of $3 \mathrm{ml} / \mathrm{min}(\mathrm{Re}=21)$ in the y,z-plane (z-axis is the flow direction). (a) A uniform pattern of the radial velocity is observed for the structure S_Ot_Op (indicated by the red box, top row), while the heterogeneity in the distribution of the radial velocity is increased by increasing the orientation angle $\phi$ (indicated by red boxes, middle row). Increasing the rotation angle $\theta$ causes connected fluid paths (indicated by the red box, bottom row). (b) Increasing the orientation angle $\phi$ diverts fluid elements radially and shortens connected fluid paths (indicated by red boxes, middle row). Increasing the rotation angle $\theta$ to $45^{\circ}$ causes longer connected fluid paths (indicated by the red box, bottom row). 
Thus, analyzing the effect of the different porous structures on the axial and radial velocity reveals that orienting the cylindrical rods generates heterogeneous regions with radial velocities of different magnitudes. This property can promote the mixing behavior of the reactors which will be further investigated in the following sections.

\subsubsection{Velocity Deformation Metrics}

Local flow features determine mixing performance, and such flow features can be identified based on local velocity gradients (Weeks \& Sposito 1998). Thus, in this study we calculate the flow number, the stretching efficiency and the finite-time Lyapunov exponent (FTLE) as flow deformation metrics, which are all based on local velocity gradients, to identify flow features. The flow number $\alpha$ (Yang \& Manas-Zloczower 1992) quantifies the extensional or rotational behavior of fluid elements, and it is defined as

$$
\alpha=\frac{|\dot{\gamma}|}{|\dot{\gamma}|+|\dot{\omega}|}
$$

with the strain rate tensor $\dot{\gamma}$

$$
\dot{\gamma}=1 / 2\left[\nabla \vec{V}+(\nabla \vec{V})^{T}\right]
$$

and the vorticity tensor $\dot{\omega}$

$$
\dot{\omega}=1 / 2\left[\nabla \vec{V}-(\nabla \vec{V})^{T}\right]
$$

A value of $\alpha=0$ corresponds to pure rotation, while $\alpha=1$ indicates pure extension. Quantifying the flow number reveals that extension is more dominant than rotation in all porous structures (see section S2 and Figure S1 in the Supplementary Information). Figure 6 depicts contours of the radial velocity normalized with the pore velocity with the iso-surface of $\alpha=0.7$ superimposed. It is observed that increasing the orientation angle $\phi$ of the cylindrical rods generates non-uniformity in the radial velocity distribution and the flow number iso-surface. However, the influence of the rotation angle $\theta$ on this non-uniformity is less pronounced. This 
non-uniform extensional behavior of the fluid elements increases the contact area between them, which will reduce the diffusion distance between fluid layers and thus facilitates mixing (Ottino 1989).
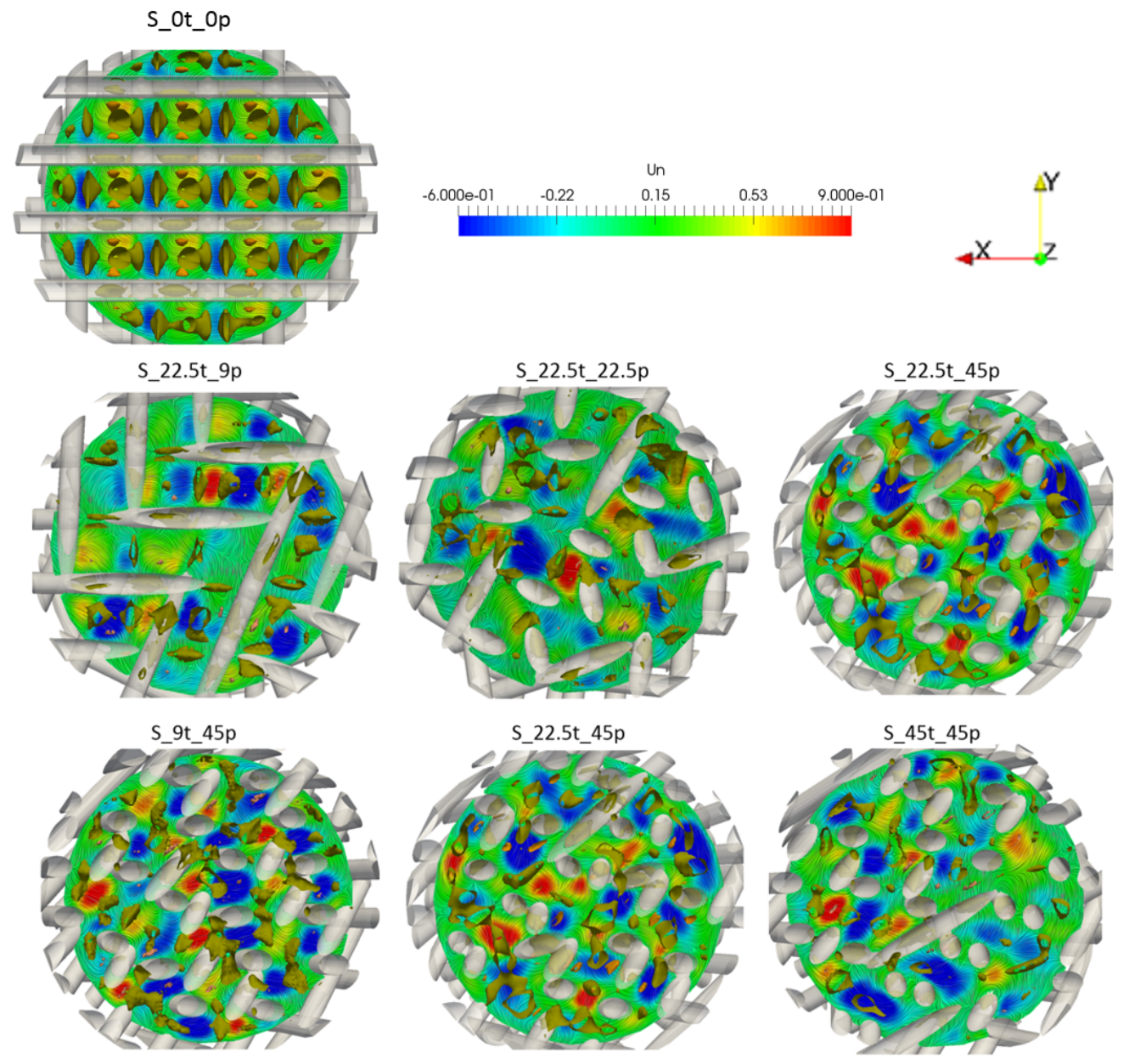

Figure 6 Contours of the radial velocity (normalized by the pore velocity) in the different WSPM extracted at the streamwise position $z=0.5 L$. Iso-surfaces of the flow number $\alpha=0.7$ are superimposed (gold color).

As the flow inside the structures is governed by extension, the stretching efficiency $e_{\lambda}$, which provides a measure of how a material line element is stretched by the fluid velocity gradient, is quantified (Ottino 1991). It is calculated as 


$$
e_{\lambda}=\frac{D \ln \lambda / D t}{(D: D)^{1 / 2}}=\frac{|m \cdot D \cdot m|}{(D: D)^{1 / 2}}
$$

where $\lambda$ is the length stretch of the material line, $D=\dot{\gamma}$, and $m$ is a unit vector tangential to the material line. $D$ has eigenvalues $\delta_{i}$ and eigenvectors $n_{i}$, and it has been shown that a maximum stretching efficiency $e_{\lambda \max }$ is obtained when the line orientation $m$ is identical to the eigenvector $n_{1}$ (Ottino 1991), and it can be quantified as

$$
e_{\lambda \max }=\frac{\delta_{1}}{(D: D)^{1 / 2}}
$$

The value of $e_{\lambda \max }$ allows to differentiate different flow types (Ottino 1989), i.e. $e_{\lambda \max }>0.77$ corresponds to pure elongation, $0.67<e_{\lambda \max }<0.72$ indicates simple shear, and $e_{\lambda \max }<$ 0.40 denotes squeezing. Figure 7 depicts the relative volume fraction of the maximum stretching efficiency (volume-weighted average of $e_{\lambda \max }$ ) extracted in all porous structures. As it can be seen, for the structure S_22.5t_45p, a larger volume fraction is dominated by elongation and a comparatively smaller volume fraction with shear flow in contrast to the other geometries. Increasing the orientation angle from $9^{\circ}$ to $22.5^{\circ}$ (S_22.5t_9p to $\mathrm{S} \_22.5 \mathrm{t} \_22.5 \mathrm{p}$ ) causes an approximately 5\% reduction in shear behavior and 3\% rise in elongation. A further increase to $45^{\circ}$ (S_22.5t_22.5p to S_22.5t_45p) decreases shear and increases elongation by $10 \%$ and $7.2 \%$ respectively. This increase of elongation in the flow field in the porous structures with higher orientation can be attributed to the larger heterogeneity in the radial velocities (Figure 5), which eventually leads to mixing improvement (de Barros et al. 2012). 


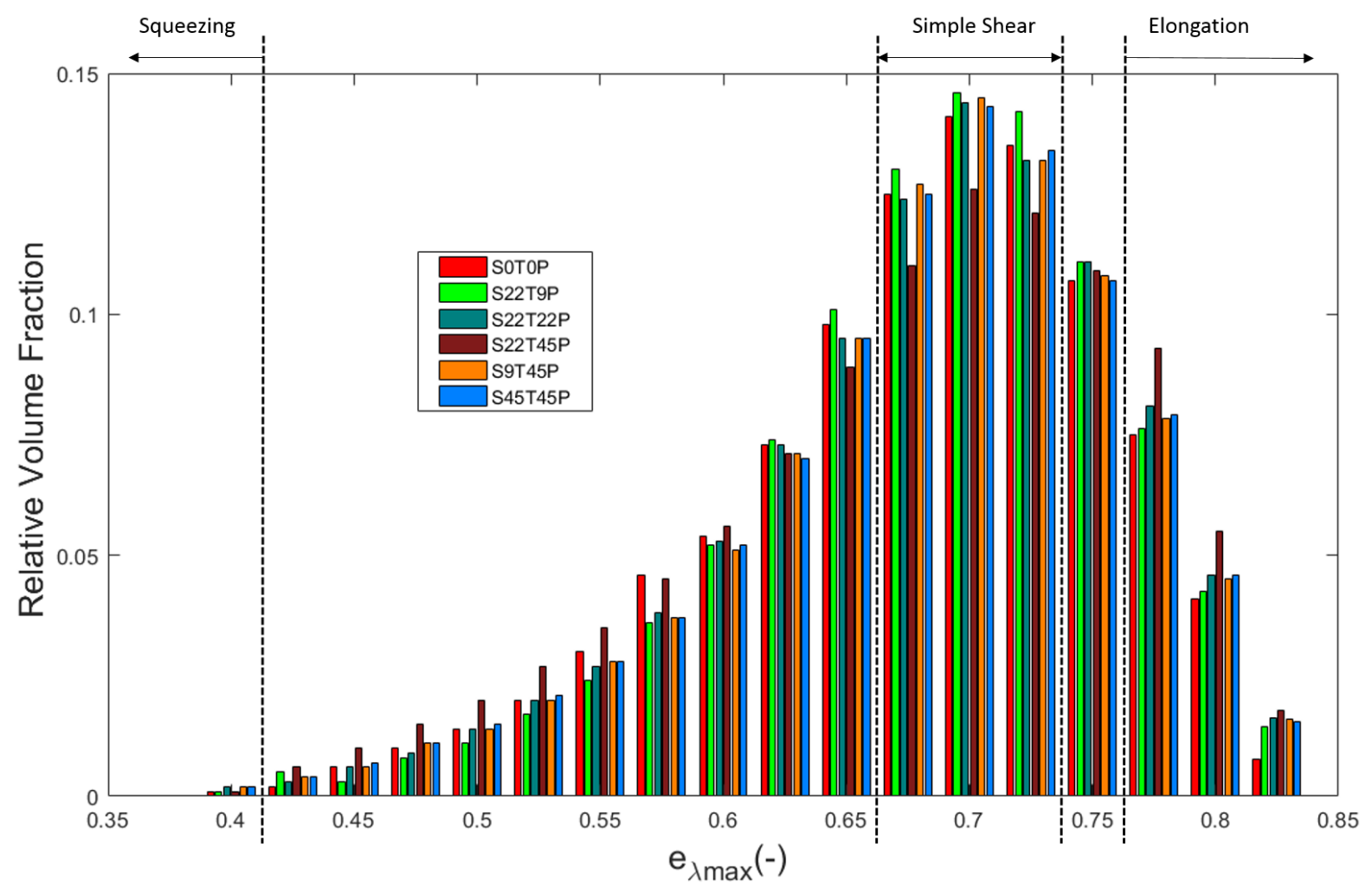

Figure7 Relative volume fraction of the maximum stretching efficiency $e_{\lambda \max }$ for all WSPM.

The dashed lines separate the different flow type regions.

Finally, the finite-time Lyapunov exponent (FTLE) is computed, which quantifies the amount of stretching between fluid particles in a given time $T$. The FTLE is quantified as the largest eigenvalue $\delta_{\text {max }}$ of the Cauchy-Green strain tensor $C_{t_{0}}^{t_{0}+T}$

$$
\operatorname{FTLE}\left(x, t_{0}, T\right)=\frac{1}{T} \ln \|A\|_{2}
$$

with

$$
\|A\|_{2}=\sqrt{\delta_{\max }\left(C_{t_{0}}^{t_{0}+T}\right)}
$$

The Cauchy-Green strain tensor is calculated by multiplying the Lagrangian deformation gradient tensor $A$ of $F_{t_{0}}^{t_{0}+T}$ with its transpose

$$
C_{t_{0}}^{t_{0}+T}=[\mathrm{A}]^{t} \cdot A
$$

with 


$$
A=\nabla F_{t_{0}}^{t_{0}+T}\left(x_{0}\right)
$$

$F_{t_{0}}^{t_{0}+T}$ denotes the flow map of fluid particles from their initial positions to their positions at time $T$.

To compute the Cauchy-Green deformation tensor, trajectories of particles seeded in each point of the grid were integrated over time with a Runge-Kutta integrator. These FTLE computations were performed using VisIt (Livermore Lawrence National Laboratory 2018) on a subdomain of the initial CFD domain. It is worth noting that Lagrangian coherent structures (LCS) can be extracted from maxima ridges of FTLE. LCS extraction has proven to be an efficient tool for the visualization of transport structures in complex fluid flow system (Lukens et al. 2010). This method is a practical way to identify groups of fluid parcels that behave similarly (coherent), and subsequently to visualize the flow skeleton. LCS represent surfaces which divide the flow structure into regions, corresponding to manifolds with minimum transverse flux. Figure 8 depicts the LCS in the structures S_0t_0p and S_22.5t_45p, where the purple color represents coherent fluid volumes, and the cyan color denotes the LCS surfaces that divide these coherent volumes. It can be observed that applying orientation in the porous structure creates more nonuniform size regions (Figure 8, column 2) as well as more tortuous fluid passages (Figure 8, column 3). Chaotic advection is promoted when the minimal transverse flux surfaces intersect at an angle (Ottino \& Wiggins 2004; Lester et al. 2016), and this behavior is more noticeable for the porous structure $S \_22.5 t \_45 p$. 


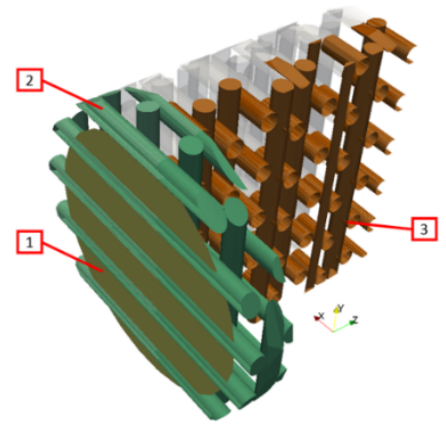

1
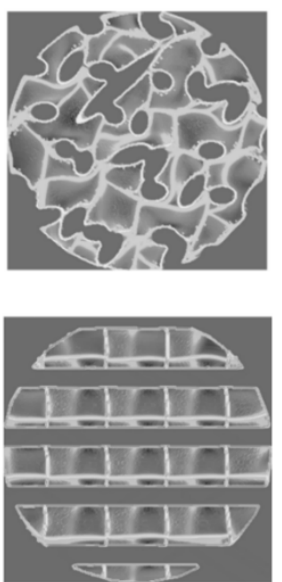

2
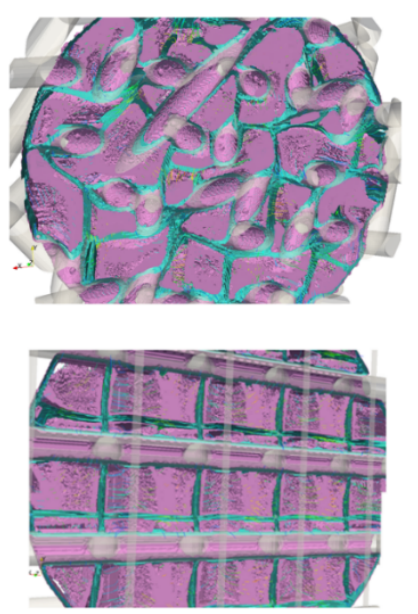

3

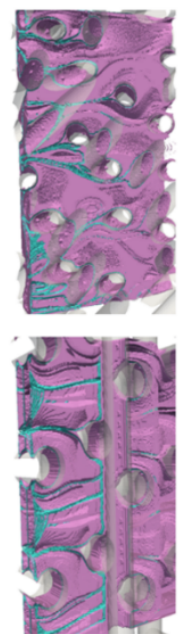

Figure 8 Illustration of Lagrangian coherent structures (LCS). The top row depicts the porous structure $S \_22.5 t \_45 p$ and the bottom row S_0t_Op. Column 1 visualizes the FTLE field for a flow rate of $3 \mathrm{ml} / \mathrm{min}$ within the two structures; column 2 depicts the radial distribution of coherent fluid volumes (purple) divided by LCS surfaces (cyan); column 3 shows the axial LCS distribution.

The FTLE analysis has also been performed for the other porous structures (see section S3 and Figure S2 in the Supplementary Information). In general, it is observed that applying rotation and orientation leads to the creation of more heterogeneous fluid passages.

To conclude the hydrodynamic characterization, the results indicate that the introduction of orientation in the micro-cylindrical rod layers increases the flow heterogeneity and shifts the flow behavior towards elongation. These features are significant for mixing enhancement, which will be quantified in the next section.

\subsection{Radial Mixing}

To characterize the radial mixing in the porous structures, the scalar transport simulations as described in section 3 were performed. Figure 9 depicts contours of the tracer concentration extracted in cross-sections of equal residence time for all WSPM and the empty tube $(t=0.5 \tau$, 
see section S4 in the Supplementary Information for details on the RTD analysis). For the empty tube, barely any radial mixing is observed, whereas the tracer plume is spread when interacting with the porous structures. As it is evident from Figure 9, the structure S_22.5t_45p results in the largest radial mixing, which is again attributed to its increased flow heterogeneity and invoked tortuous fluid paths.

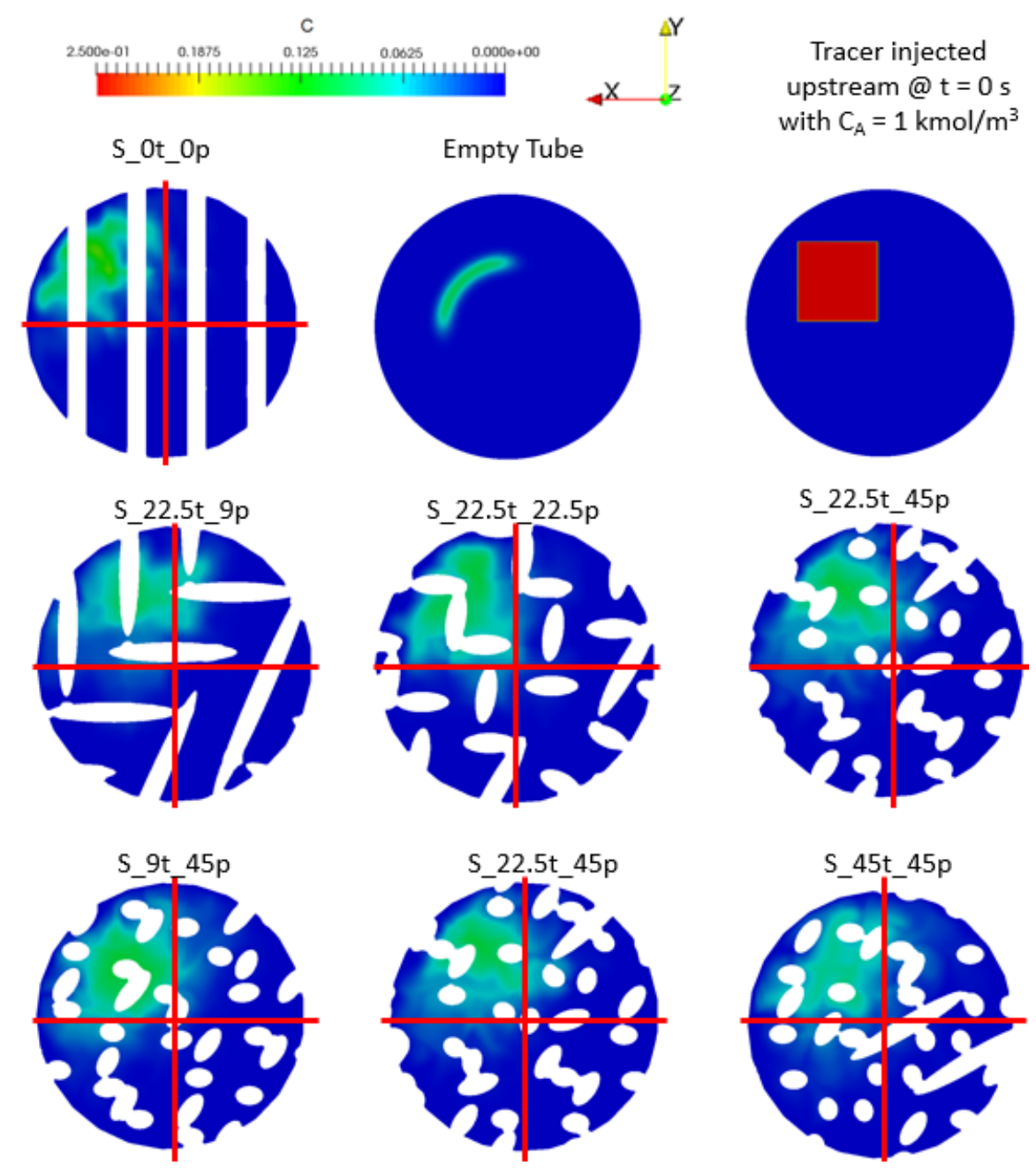

Figure 9 Contours of the tracer distribution extracted in cross-sections of equal residence time $(t=0.5 \tau)$ for all WSPM and the empty tube. All structures enhance the radial mixing of the tracer in contrast to the empty tube. 
To quantitatively evaluate the mixing performance of the structures the intensity of segregation, $I_{S}$, is calculated ((Danckwerts 1952) and (Bothe 2010))

$$
I_{s}=\frac{S^{2}}{S_{\max }^{2}}
$$

where $I_{S}=0$ denotes complete mixing, and $I_{S}=1$ complete segregation, with

$$
\begin{gathered}
S^{2}=\frac{1}{N-1} \sum_{i=1}^{N}\left(C_{A, i}-\bar{C}_{A}\right)^{2} \\
S_{\max }^{2}=\bar{C}_{A}\left(1-\bar{C}_{A}\right)
\end{gathered}
$$

and

$$
\bar{C}_{A}=\frac{1}{N} \sum_{i=1}^{N} C_{A, i}
$$

where $\bar{C}_{A}$ is the average concentration, $C_{A, i}$ is the concentration in each computational cell and $N$ is the total number of computational cells in each cross-section.

Figure 10 shows the resulting $I_{S}$ values, together with the pressure drop across the different WSPM and an empty tube. It is observed that inserting the WSPM into a tubular reactor increases the pressure drop while it also considerably decreases the intensity of segregation, i.e. significantly improves the mixing. This trend is most pronounced for the structure $\mathrm{S} \_22.5 \mathrm{t} \_45 \mathrm{p}$. For example, S_22.5t_45p exhibits $75 \%$ lower intensity of segregation in comparison to S_0t_0p while the pressure drop is only increased by $18 \%$. The mixing improvement is linked to the heterogeneous flow structures and associated chaotic advection visualized by the FTLE analysis, and it is mainly linked to the orientation angle $\phi$ of the cylindrical rods. 

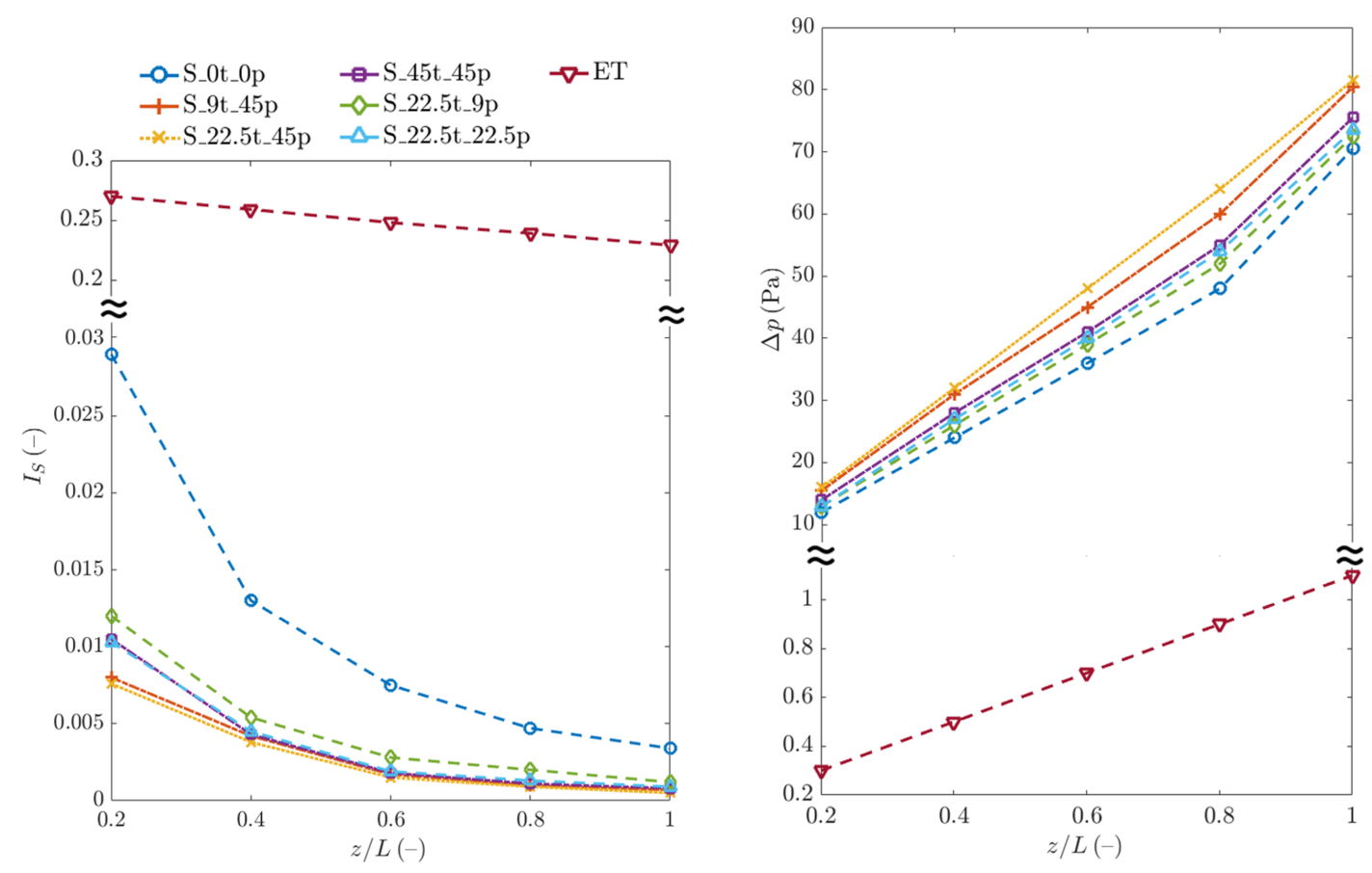

Figure 10 Profiles of the intensity of segregation $I_{S}$ (left) and the pressure drop $\Delta p$ (right) across the WSPM and an empty tube.

Furthermore, we compared the coefficient of variation $(\mathrm{CoV})$, defined as

$$
\operatorname{CoV}=\frac{\sigma}{\bar{C}_{A}}=\frac{\sqrt{\frac{1}{N-1} \sum_{i=1}^{N}\left(C_{A, i}-\bar{C}_{A}\right)^{2}}}{\bar{C}_{A}}
$$

and extracted at $z=L$ of the S_0t_0p and S_22.5t_45p structures with data reported in literature for metal foams of 20, 30 and 45 PPI (Hutter et al. 2010). As it can be seen in Figure 11, the WSPM in this study provide lower CoV values (enhanced homogenization performance) than the random metal foams in laminar flow $(\operatorname{Re}<100)$. 


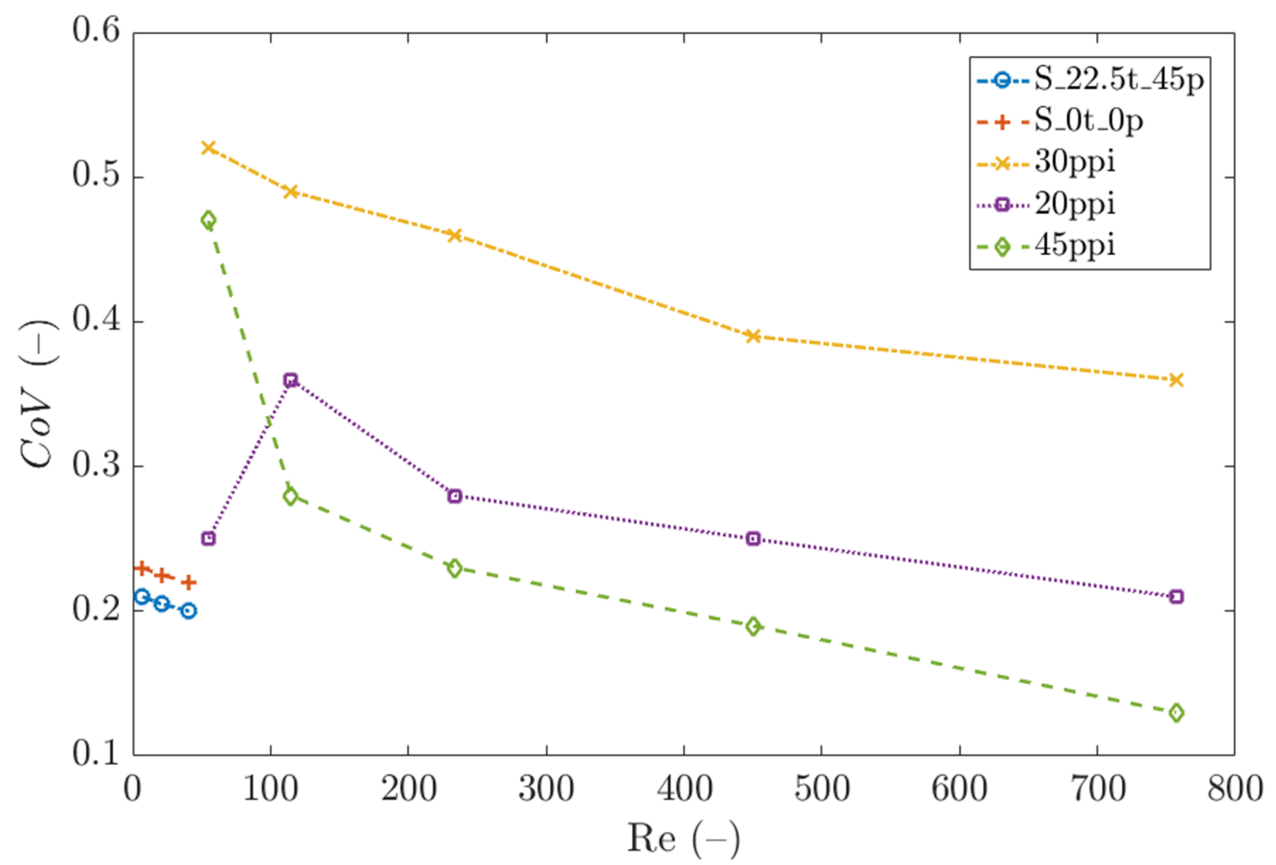

Figure 11 Coefficient of variation (CoV) for the S_0t_Op and S_22.5t_45p structures compared with literature data for three metal foams of 20, 30, and 45 PPI (Hutter et al. 2010). The Reynolds number is calculated with the empty tube diameter and the bulk velocity.

\section{Conclusions}

In this work, we studied the hydrodynamics and mixing performance of milli-scale tubular reactors equipped with well-structured porous media (WSPM) by the aid of CFD. Six different configurations of WSPM were considered, which differ in the shift of individual cylindrical rods between layers, the rotation of rod layers around the main flow direction, and the orientation of rod layers against the main flow direction.

The hydrodynamic analysis revealed that the value of the orientation angle $\phi$ of the cylindrical rods affects the flow heterogeneity and the radial velocity magnitudes. Secondly, the flow deformation metrices confirmed that the strain rate is more dominant than the vorticity in the WSPM. And applying larger orientation angle value creates more elongation in the flow field which was attributed to the increase of heterogeneity in the radial velocities. Thus, the 
hydrodynamic characterization revealed that introducing the orientation on the microcylindrical rod layers impacts greatly the resulting flow pattern.

Scalar simulations were then performed to quantify the impact of these observed effects on radial mixing. An enhancement of radial mixing was found for all WSPM in comparison to an empty tube, and the mixing improvement was linked to the heterogeneous flow structures and associated chaotic advection. In addition, it was observed that the structure S_22.5t_45p provided the best mixing, with a decrease of $75 \%$ in the intensity of segregation compared to the structure S_0t_0p. This decrease has been achieved with a minor increase $(18 \%)$ in pressure drop.

This study shows the potential of using WSPM in tubular reactors as a scale-up approach and the link of geometric parameters with their performance. Specifically, we identified that applying orientation on the rod layers ( angle $\phi$ ) with respect to the plane normal to the main flow direction enhances the mixing in the milli-tubular reactor. A geometrical analysis reveals the importance of the angle $\phi$ on creating flow features essential for promoting mixing. And altering $\phi$ influences the alignment and connection of individual pores inside the structure in such a way that it leads to radial deflection of fluid elements and non-uniform fluid element distribution.

\section{Notation}

Roman Symbols

A Gradient of the flow map tensor [-]

$A^{t} \quad$ Transpose of the gradient of the flow map tensor [-]

$\|A\|_{2} \quad$ Largest eigenvalue of the Cauchy-Green strain tensor [-]

$C_{A} \quad$ Concentration $\left[\mathrm{mol} . \mathrm{m}^{-3}\right]$

$\bar{C}_{A} \quad$ Average concentration $\left[\mathrm{mol} . \mathrm{m}^{-3}\right]$

$C_{A, \text { in }}$ Inlet concentration [mol.m $\left.\mathrm{m}^{-3}\right]$ 
$C_{A, \text { out }}$ Outlet concentration $\left[\mathrm{mol} . \mathrm{m}^{-3}\right]$

$C_{t_{0}}^{t_{0}+T}$ Cauchy-Green strain tensor [-]

$D_{A} \quad$ Diffusivity $\left[\mathrm{m}^{2} \cdot \mathrm{s}^{-1}\right]$

E Residence-time distribution function $\left[\mathrm{s}^{-1}\right]$

$\vec{V} \quad$ Velocity $\left[\mathrm{m} . \mathrm{s}^{-1}\right]$

$\mathrm{p} \quad$ Pressure $[\mathrm{Pa}]$

Re Reynolds number [-]

$\mathrm{t} \quad$ Time [s]

$e_{\lambda} \quad$ Stretching efficiency [-]

$e_{\lambda \max }$ Maximum stretching efficiency [-]

M, N Model parameters [-]

$\mathrm{S}^{2} \quad$ Segregation $\left[\mathrm{mol} . \mathrm{m}^{-3}\right]^{2}$

$\mathrm{I}_{\mathrm{s}} \quad$ Intensity of segregation [-]

$\mathrm{n} \quad$ Eigenvector

Greek Symbols

$\alpha \quad$ Flow number [-]

$\omega \quad$ Vorticity $\left[\mathrm{s}^{-1}\right]$

$\gamma \quad$ Strain rate $\left[\mathrm{s}^{-1}\right]$

$\sigma \quad$ Variance [-]

$v \quad$ Kinematic viscosity $\left[\mathrm{m}^{2} \cdot \mathrm{s}^{-1}\right]$

$\theta \quad$ Rotation around $\mathrm{z}$-axis $\left[{ }^{\circ}\right]$

$\phi \quad$ Orientation against $\mathrm{z}$-axis $\left[^{\circ}\right]$

$\lambda \quad$ Length stretch of material line

$\delta \quad$ Eigenvalue 


$\begin{array}{ll}\nabla & \text { Del operato } \\ \nabla^{2} & \text { Laplace op } \\ \text { Subscripts } \\ \text { max } & \text { Maximum } \\ \text { min } & \text { Minimum } \\ \text { in } & \text { Inlet } \\ \text { out } & \text { Outlet } \\ \text { i } & \text { Sequence }\end{array}$

Abbreviations

CFD Computational Fluid Dynamics

COV Coefficient of Variation

FTLE Finite Time Lyapunov Exponent

LCS Lagrangian Coherent Structure

LIC Linear Integral Convolution

RTD Residence Time Distribution

WSPM Well-Structured Porous Medium

\section{Acknowledgments}

S. K. acknowledges financial support from FWO (Odysseus II Grant No. G0E6314N).

\section{References}

de Barros, F.P.J., Dentz, M.J., Koch, J., et al., 2012. Flow topology and scalar mixing in spatially heterogeneous flow fields. Geophysical Research Letters, 39(8), L08404.

Boomsma, K. \& Poulikakos, D., 2002. The Effects of Compression and Pore Size Variations 
on the Liquid Flow Characteristics in Metal Foams. Journal of Fluids Engineering, 124(1), p.263.

Bothe, D., 2010. Evaluating the Quality of a Mixture: Degree of Homogeneity and Scale of Segregation. In Springer, Berlin, Heidelberg, pp. 17-35.

Busse, C., Freund, H. \& Schwieger, W., 2018. Intensification of heat transfer in catalytic reactors by additively manufactured periodic open cellular structures (POCS). Chemical Engineering and Processing - Process Intensification, 124, pp.199-214.

Code_aster, SALOME Platform July 2018. https://www.salome-platform.org/.

Danckwerts, P. V., 1952. The definition and measurement of some characteristics of mixtures. Applied Scientific Research, 3(4), pp.279-296.

Giani, L., Groppi, G. \& Tronconi, E., 2005a. Heat Transfer Characterization of Metallic Foams. Industrial \& Engineering Chemistry Research, 44(24), pp.9078-9085.

Giani, L., Groppi, G. \& Tronconi, E., 2005b. Mass-Transfer Characterization of Metallic Foams as Supports for Structured Catalysts. Industrial \& Engineering Chemistry Research, 44(14), pp.4993-5002.

Häfeli, R., Ruegg, O., Altheimer, M., et al., 2016. Investigation of emulsification in static mixers by optical measurement techniques using refractive index matching. Chemical Engineering Science, 143, pp.86-98.

Hall, M.J. \& Hiatt, J.P., 1994. Exit flows from highly porous media. Physics of Fluids, 6(2), pp.469-479.

Haller, G., 2001. Distinguished material surfaces and coherent structures in three-dimensional fluid flows. Physica D: Nonlinear Phenomena, 149(4), pp.248-277.

Harnby, N., Edwards, M.F. \& Nienow, A.W., 1997. Mixing in the process industries, Butterworth-Heinemann.

Hutter, C., Zenklusen, A., Lang, R., et al., 2011a. Axial dispersion in metal foams and streamwise-periodic porous media. Chemical Engineering Science, 66(6), pp.1132-1141. 
Hutter, C., Zenklusen, A., Kuhn, S., et al., 2011b. Large eddy simulation of flow through a streamwise-periodic structure. Chemical Engineering Science, 66(3), pp.519-529.

Hutter, C. et al., 2010. Scalar transport in a milli-scale metal foam reactor. Chemical Engineering Science, 65(10), pp.3169-3178.

Huu, T.T., Lacroix, M, Pham Huu, C., et al., 2009. Towards a more realistic modeling of solid foam: Use of the pentagonal dodecahedron geometry. Chemical Engineering Science, 64(24), pp.5131-5142.

Inayat, A., Schwerdtfeger, J., Freund, H., et al., 2011. Periodic open-cell foams: Pressure drop measurements and modeling of an ideal tetrakaidecahedra packing. Chemical Engineering Science, 66(12), pp.2758-2763.

Klumpp, M., Inayat, A., Schwerdtfeger, J., et al., 2014. Periodic open cellular structures with ideal cubic cell geometry: Effect of porosity and cell orientation on pressure drop behavior. Chemical Engineering Journal, 242, pp.364-378.

Krishnan, S., Murthy, J.Y. \& Garimella, S. V., 2006. Direct Simulation of Transport in OpenCell Metal Foam. Journal of Heat Transfer, 128(8), p.793.

Kumar, P. \& Topin, F., 2014. Investigation of fluid flow properties in open cell foams: Darcy and weak inertia regimes. Chemical Engineering Science, 116, pp.793-805.

Lacroix, M., Nguyen, P., Schweich, D., et al., 2007. Pressure drop measurements and modeling on SiC foams. Chemical Engineering Science, 62(12), pp.3259-3267.

Lämmermann, M., Horak, G., Schwieger, W., et al., 2018. Periodic open cellular structures (POCS) for intensification of multiphase reactors: Liquid holdup and two-phase pressure drop. Chemical Engineering and Processing - Process Intensification, 126, pp.178-189.

Lämmermann, M., Schwieger, W. \& Freund, H., 2016. Experimental investigation of gasliquid distribution in periodic open cellular structures as potential catalyst supports. Catalysis Today, 273, pp.161-171.

Lang, C., Sécordel, X. \& Courson, C., 2017. Copper-Based Water Gas Shift Catalysts for 
Hydrogen Rich Syngas Production from Biomass Steam Gasification. Energy \& Fuels, 31(11), pp.12932-12941.

Lester, D.R., Dentz, M. \& Le Borgne, T., 2016. Chaotic mixing in three-dimensional porous media. Journal of Fluid Mechanics, 803, pp.144-174.

Levenspiel, O., 1999. Chemical reaction engineering, Wiley.

Livermore Lawrence National Laboratory, VisIt Feb 2019, https://wci.llnl.gov/simulation/computer-codes/visit/.

Lukens, S., Yang, X. \& Fauci, L., 2010. Using Lagrangian coherent structures to analyze fluid mixing by cilia. Chaos: An Interdisciplinary Journal of Nonlinear Science, 20(1), p.17511.

Ortega-Casanova, J., 2017. Application of CFD on the optimization by response surface methodology of a micromixing unit and its use as a chemical microreactor. Chemical Engineering and Processing: Process Intensification, 117, pp.18-26.

Ottino, J.M., 1989. The kinematics of mixing : stretching, chaos, and transport, Cambridge University Press.

Ottino, J.M., 1991. Unity and diversity in mixing: Stretching, diffusion, breakup, and aggregation in chaotic flows. Physics of Fluids A: Fluid Dynamics, 3(5), pp.1417-1430.

Ottino, J.M. \& Wiggins, S., 2004. Introduction: mixing in microfluidics. Philosophical transactions. Series A, Mathematical, physical, and engineering sciences, 362(1818), pp.923-35.

Podyacheva, O.Y., Ketov, A., Ismagilov, Z., et al., 1997. Metal foam supported perovskite catalysts. Reaction Kinetics and Catalysis Letters, 60(2), pp.243-250.

Potdar, Thomassen, L.C.J. \& Kuhn, S., 2017. Designed porous milli-scale reactors with enhanced interfacial mass transfer in two-phase flows. Reaction Chemistry \& Engineering, 2(2), pp.137-148.

Potdar, A., Thomassen, L.C.J. \& Kuhn, S., 2019a. Scalability of 3D printed structured porous 
milli-scale reactors. Chemical Engineering Journal, 363, pp.337-348.

Potdar, A., Thomassen, L.C.J. \& Kuhn, S., 2019b. Structured Porous Millireactors for LiquidLiquid Chemical Reactions. Chemie Ingenieur Technik, 91, pp592-601.

Rahimi, M., Akbari, M., Parsamoghadam, M., et al., 2015. CFD study on effect of channel confluence angle on fluid flow pattern in asymmetrical shaped microchannels. Computers \& Chemical Engineering, 73, pp.172-182.

Razza, S., Heidig, E.,Bianchi, E., et al., 2016. Heat transfer performance of structured catalytic reactors packed with metal foam supports: Influence of wall coupling. Catalysis Today, 273, pp.187-195.

Serres, M., Schweich, D., Vidal, V., et al., 2018. Liquid residence time distribution of multiphase horizontal flow in packed bed milli-channel: Spherical beads versus open cell solid foams. Chemical Engineering Science, 190, pp.149-163.

Serres, M., Zanota, M., Philippe, R., et al., 2016. On the stability of Taylor bubbles inside a confined highly porous medium. International Journal of Multiphase Flow, 85, pp.157163.

Suranani, S., Maralla, Y., Gaikwad, S., et al., 2018. Process intensification using corning ${ }^{\circledR}$ advanced-flow ${ }^{\mathrm{TM}}$ reactor for continuous flow synthesis of biodiesel from fresh oil and used cooking oil. Chemical Engineering and Processing - Process Intensification, 126, pp.62-73.

Della Torre, A., Montenegro, G., Tabor, G., et al., 2014. CFD characterization of flow regimes inside open cell foam substrates. International Journal of Heat and Fluid Flow, 50, pp. $72-82$.

Della Torre, A., Lucci, F., Montenegro, G., et al., 2016. CFD modeling of catalytic reactions in open-cell foam substrates. Computers \& Chemical Engineering, 92, pp.55-63.

Tourvieille, J.-N., Philippe, R. \& de Bellefon, C., 2015a. Milli-channel with metal foams under an applied gas-liquid periodic flow: External mass transfer performance and 
pressure drop. Chemical Engineering Journal, 267, pp.332-346.

Tourvieille, J.-N., Philippe, R. \& de Bellefon, C., 2015b. Milli-channel with metal foams under an applied gas-liquid periodic flow: Flow patterns, residence time distribution and pulsing properties. Chemical Engineering Science, 126, pp.406-426.

Twigg, M. V \& Richardson, J.T., 2007. Fundamentals and Applications of Structured Ceramic Foam Catalysts. Industrial \& Engineering Chemistry Research, 46(12), pp.4166-4177.

Versteeg, H.K. (Henk K. \& Malalasekera, W. (Weeratunge), 2007. An introduction to computational fluid dynamics : the finite volume method, Pearson Education Ltd.

Weeks, S.W. \& Sposito, G., 1998. Mixing and stretching efficiency in steady and unsteady groundwater flows. Water Resources Research, 34(12), pp.3315-3322.

Weller, H., OpenFOAM | Free CFD Software | The OpenFOAM Foundation July 2019. https://openfoam.org/.

Woldemariam, M., Filimonov, R., Purtonen, T., et al., 2016. Mixing performance evaluation of additive manufactured milli-scale reactors. Chemical Engineering Science, 152, pp.26-34.

Yang, H.-H. \& Manas-Zloczower, I., 1992. Flow field analysis of the kneading disc region in a co-rotating twin screw extruder. Polymer Engineering and Science, 32(19), pp.14111417.

Zhang, J., Wang, K., Teixeira, A., et al., 2017. Design and Scaling Up of Microchemical Systems: A Review. Annual Review of Chemical and Biomolecular Engineering, 8(1), pp. $285-305$. 